\title{
Effect of early vitamin D supplementation on asthma and the possible mechanisms
}

\author{
X. Chen' ${ }^{1}$, S.Q. Rao ${ }^{2}$, B.H. Gao ${ }^{3}$ and Z.Q. Jiang ${ }^{1}$ \\ 1'Department of Nutrition, College of Public Health, Sun Yat-Sen University, \\ Guangzhou, China \\ ${ }^{2}$ Department of Pediatrics, Liwan Hospital, Guangzhou Medical College, \\ Guangzhou, China \\ ${ }^{3}$ Department of Children's Health, Huzhong Hospital, Guangzhou, China \\ Corresponding author: X. Chen \\ E-mail: chenxiangsci@163.com
}

Genet. Mol. Res. 14 (4): 14136-14143 (2015)

Received June 17, 2015

Accepted August 27, 2015

Published October 29, 2015

DOI http://dx.doi.org/10.4238/2015.October.29.35

\begin{abstract}
Asthma is a chronic inflammatory disease of the airways with variable airflow obstruction and bronchial hyper-responsiveness. It is believed that Th2-derived cytokines orchestrate the asthmatic response and the maintenance of the balance of Th1/Th2 plays a crucial role in prevention of asthma. Moreover, $1,25(\mathrm{OH})_{2} \mathrm{D} 3$, the biologically activate form of vitamin $\mathrm{D}$, was reported with an almost opposite role in prevention and treatment of asthma. Therefore, in this study, we elucidated the evaluations of in vivo anti-asthma effects when treated with different doses of vitamin $D$ and validated the relationship between vitamin $D$ and asthma. Our data demonstrated that intervention with the appropriate dose of $1,25(\mathrm{OH})_{2} \mathrm{D} 3$ in early life could improve pulmonary function and reduce eosinophil cell infiltration in the airways of rat asthma models. However, overdose might play a detrimental effect. Its mechanism may correlate with the effect of $1,25(\mathrm{OH})_{2} \mathrm{D} 3$ on interleukin (IL)-4, IL-12, IL-13, interferon-y, and ovalbumin-specific immunoglobulin $\mathrm{E}$ secretion and the expression of p-JAK1/p-STAT6/SOCS5.
\end{abstract}

Key words: Vitamin D; Infant; Weaning rats; p-JAK1/p-STAT6/SOCS5 


\section{INTRODUCTION}

Bronchial asthma is a chronic airway inflammatory disease involving many inflammatory cells (Hiemstra et al., 2015; Liu et al., 2015; Pelaia et al., 2015). An imbalanced Th1/Th2 ratio that induces the production of Th2 cytokines such as interleukin (IL)-4 and IL-13 and inhibits Th1 cytokines such as interferon (IFN)- $\mathrm{Y}$, is the key factor of asthma (Truyen et al., 2006; He et al., 2014). Recently, asthma has become one of the most serious and common respiratory diseases and its incidence has climbed markedly in the past two decades. Given that asthma results in an economic and social burden that is both substantial and increasing, research on the prevention and treatment of asthma is becoming more and more urgent (Camargo et al., 2007).

In addition to its roles in bone and calcium metabolism and cell proliferation and differentiation, $1,25(\mathrm{OH})_{2} \mathrm{D} 3$, the biologically activate form of vitamin $\mathrm{D}$, has recently been confirmed to be involved in the modulation of asthma (Chambers et al., 2014; Tian et al., 2014). However, thus far, the role of $1,25(\mathrm{OH})_{2} \mathrm{D} 3$ in Th2-mediated allergic asthma, especially in infants, was still in dispute (Adorini, 2002; Van Overtvelt et al., 2008; Erkkola et al., 2009). Most epidemiologic studies showed that vitamin D supplementation has a protective role against wheezing illnesses in adults and infants (Devereux et al., 2007; Zhou et al., 2015), while some studies showed an adverse effect of vitamin $D$ on asthma in infants.

Therefore, our objective was to investigate the relationship between vitamin $D$ and asthma, by using different doses of vitamin $D$ in both newborn and weaning rats and to research the possible mechanisms involved to offer new ideas, a basis for the prevention and treatment of asthma, and a reasonable use of vitamin D.

\section{MATERIAL AND METHODS}

\section{Ethics statement}

The study was carried out in compliance with the guidance of the Animal Care Committee of Sun Yat-Sen University. The study protocol was approved by the Ethics Committee of Sun YatSen University. All surgery was performed under sodium pentobarbital anesthesia, and all efforts were made to minimize suffering.

\section{Animals and experiment groups}

Fifty-six 3-week-old male Wistar rats were purchased from the Sun Yat-Sen University Laboratory Animal Center (Guangzhou, China). All mice were maintained in the laboratory for animal experimentation in a specific pathogen-free environment, in laminar airflow conditions at a temperature of $25^{\circ} \pm 2^{\circ} \mathrm{C}$ under a 12-h light-dark cycle. All animals had free access to standard laboratory mouse food and water.

Rats were randomly divided into seven groups $(N=8)$ : normal group, vitamin $D$ - no allergy group, asthma group, control group treated with physiological saline, low-dose vitamin D group, middle-dose vitamin D group, and high-dose vitamin D group.

Vitamin $\mathrm{D}$ at $4,1,4$, and $10 \mu \mathrm{g} / \mathrm{kg}$ were administered intraperitoneally in the vitamin D no allergy group and in the latter three groups once and twice a day, respectively. All rats except those in the normal group and vitamin D - no allergy group, were injected in the abdomen with 
$1 \mathrm{~mL}$ mixture (10\% ovalbumin) OVA $100 \mathrm{mg}$, aluminum hydroxide $200 \mathrm{mg}$ ) at day 6 and $1 \mathrm{~mL}$ mixture (1\% OVA $10 \mathrm{mg})$ at day 13, and then received an atomization inhalation with $5 \%$ daily ovalbumin for six consecutive days from day 22. The normal group and vitamin D - no allergy group received physiological saline at the same times, and then atomization inhalation with physiological saline. Twenty-four hours after the challenge, all the rats were anesthetized and killed, chests were opened, and lobi medius pulmonis of the right lung was removed rapidly.

\section{Measurements}

The tissues were fixed by $4 \%$ formaldehyde liquid, dehydrated by different concentrations of ethanol, embedded, sliced, and then dyed by hematoxylin-eosin (HE). Electron microscopy was used to evaluate the airway inflammation and blood and bronchoalveolar lavage fluid (BALF) were collected to examine the total cell number and eosinophil percentage.

The supernatants of blood and BALF were assessed to detect the concentrations of IL4, IL-12, IL-13, IFN-y, and OVA-specific IgE (OVAslgE) by using ELISA kits (Shunhe Biotech, Shanghai, China).

The proteins related to IL-4/STAT6/SOCS5 in lung tissues were detected by western blot. Lung tissues were lysed in tissue-lysis buffer. Equal quantities of protein were separated by $10 \%$ SDS-polyacrylamide gels and transferred to polyvinylidene fluoride (PVDF) membranes. The membranes were blocked with $5 \%$ non-fat milk for $2 \mathrm{~h}$ and incubated overnight with primary antibodies anti-p-JAK1, anti-JAK1, anti-p-STAT6, anti-STAT6, anti-p-STAT4, anti-STAT4, antiSOCS5, and anti-GAPDH (1:1000; Cell Signaling Technology). After being washed in TBS-T three times, PVDF membranes were incubated with horseradish peroxidase-conjugated goat antirabbit secondary antibody at a 1:5000 dilution for $2 \mathrm{~h}$. Immunocomplexes were visualized using chemiluminescence (GE, USA) following the manufacturer protocol.

\section{Statistical analyses}

All data are reported as means \pm standard deviation, and all statistical analyses were performed using SPSS version 17.0 (SPSS Inc., Chicago, IL, USA). Differences in measurements were compared using one-way analysis of variance (ANOVA), and the Fisher least-significant difference method was employed to compare the means between groups. A P value $<0.05$ was considered to be statistically significant.

\section{RESULTS}

\section{Effect of vitamin D on airway inflammation}

\section{HE staining of lung tissue}

Normal group and vitamin D - no allergy group: No obvious inflammatory cell infiltration from the bronchus of lung tissues and surroundings in rats; the wall was intact; the thickness of wall and smooth muscle were normal without congestion and edema in the tissue mucosa; the alveolar septum was narrow.

Model group and control group: Many inflammatory cells including eosinophilic granulocytes and lymphocytes, infiltration in bronchial wall around blood vessels, and bronchus 
with bronchial mucosal edema and congestion, thickening, epithelial exfoliation, microvascular leakage, increased secretion within lumen stromal cell hyperplasia, widening of alveolar septum (Martin et al., 2003).

Low-dose and middle-dose vitamin D groups: The thickness of smooth muscle wall decreased compared to that of the model group; edema, congestion of bronchial mucosa, and eosinophilic granulocyte and other inflammatory cell infiltration from lung tissue also decreased, the alveolar lumen widened.

High-dose vitamin D group: Compared with the model group, more inflammatory cells, infiltration in bronchial wall around blood vessel and bronchus, with more severe bronchial mucosal edema and congestion were observed (Matheu et al., 2003); thickening of the smooth muscle wall and widening of the alveolar septum were also observed (Figure 1).
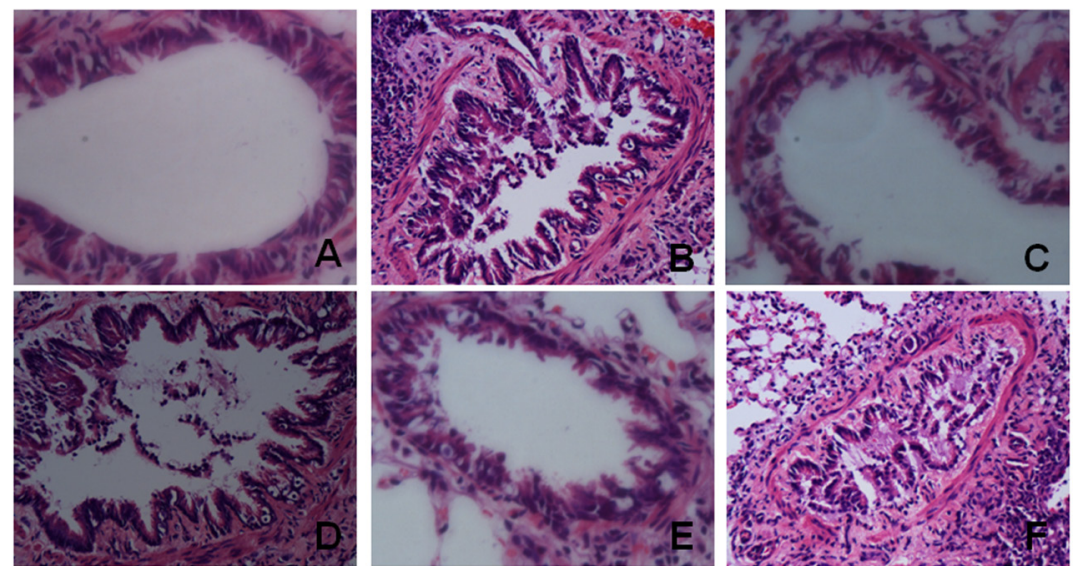

Figure 1. Effect of vitamin D on airway inflammation. A. Normal group. B. Vitamin D-no allergy group. C. Model group. D. Normal saline control group. E. Low-dose group. F. Middle-dose group. G. High-dose group.

\section{Effect of vitamin D on the total cell number and eosinophil count}

In the middle-dose and low-dose vitamin D groups, the total cell number and eosinophil percentage were lower than that in the control group $(P<0.05)$. Compared with the low-dose vitamin $\mathrm{D}$ group, the middle-dose vitamin $\mathrm{D}$ group had decreased total cell number and eosinophil percentage $(P<0.05)$, and in the high-dose vitamin $D$ group, the total cell number and eosinophil percentage were higher than that in the control group $(P<0.05$; Table 1$)$.

Table 1. Total number of cells and classification count of bronchoalveolar lavage fluid in each group.

\begin{tabular}{llccccc}
\hline Group & N & TWBC $\left(\times 10^{5} / \mathrm{mL}\right)$ & Eosinocyte $(\%)$ & Lymphomonocyte $(\%)$ & Lymphocyte $(\%)$ & Neutrophil $(\%)$ \\
\hline Normal group & 8 & $5.98 \pm 1.67$ & $1.44 \pm 0.78$ & $64.45 \pm 11.62$ & $10.31 \pm 2.45$ & $23.81 \pm 12.12$ \\
No sensitization & 8 & $8.51 \pm 1.93$ & $2.06 \pm 1.70$ & $64.88 \pm 15.73$ & $13.25 \pm 6.29^{\star}$ & $19.81 \pm 11.44$ \\
Model group & 8 & $25.34 \pm 4.28^{\star}$ & $17.81 \pm 6.89^{\star}$ & $55.31 \pm 7.21$ & $16.25 \pm 4.48$ & $10.63 \pm 5.69^{*}$ \\
Control group & 8 & $25.52 \pm 6.95^{\star}$ & $16.25 \pm 6.80^{\star}$ & $52.06 \pm 9.86^{*}$ & $20.12 \pm 8.35^{\star}$ & $13.44 \pm 6.21^{*}$ \\
VDL group & 8 & $17.24 \pm 3.3^{\Delta}$ & $15.00 \pm 5.70$ & $52.25 \pm 7.29$ & $17.38 \pm 2.43^{\star}$ & $16.00 \pm 9.79$ \\
VDM group & 8 & $9.31 \pm 3.37^{\Delta}$ & $8.89 \pm 3.66^{\Delta \Delta}$ & $57.06 \pm 8.70$ & $16.19 \pm 3.24$ & $17.25 \pm 8.70$ \\
VDH group & 8 & $45.10 \pm 15.75^{\Delta}$ & $25.88 \pm 5.57^{\Delta}$ & $42.88 \pm 4.68^{\Delta}$ & $20.19 \pm 3.38^{\Delta}$ & $11.06 \pm 6.09$ \\
\hline
\end{tabular}

${ }^{*}$ Compared with normal group $(\mathrm{P}<0.05),{ }^{\Delta}$ compared with control group $(\mathrm{P}<0.05),{ }^{\Delta}$ compared with low-dose groups $(\mathrm{P}<$ 0.05). VDL, VDM, VDH = low-dose, middle-dose, and high-dose vitamin D (VD). 


\section{Effect of vitamin D on Th1/Th2}

In the middle-dose and low-dose vitamin D groups, the IL-4, IL-13, OVAsigE, and IL-4/ INF- $\gamma$ levels in supernatants of blood and BALF were lower than those in the control group $(\mathrm{P}<$ 0.05). The IL-12 and INF-y levels in the supernatants of blood and BALF were higher than those in the asthma group $(P<0.05)$. Compared with the low-dose vitamin $D$ group, the middle-dose vitamin D group had reduced IL-4 and IL-4/INF- $y$ levels in the supernatants of blood, and reduced $\mathrm{IL}-13$ and OVAslgE levels in the supernatants of blood and BALF $(P<0.05)$. In the high-dose vitamin D group, the IL-4 level in the supernatants of BALF and the IL-4/INF- $\gamma$ in the supernatants of blood and BALF were lower than those in the asthma group $(P<0.05$; Tables 2-5).

Table 2. Levels of IL-4, INF- $y$, and IL-4/INF- $y$ in serum of rats in each group (means \pm SD).

\begin{tabular}{lllcc}
\hline Group & $\mathrm{N}$ & $\mathrm{IL}-4(\mathrm{pg} / \mathrm{mL})$ & $\mathrm{INF}-\mathrm{Y}(\mathrm{pg} / \mathrm{mL})$ & $\mathrm{IL}-4 / \mathrm{INF}-\mathrm{Y}$ \\
\hline Normal group & 8 & $0.62 \pm 0.54$ & $79.7 \pm 10.08$ & $0.007 \pm 0.006$ \\
VD - no allergy group & 8 & $0.92 \pm 0.46$ & $78.10 \pm 8.58$ & $0.012 \pm 0.006$ \\
Model group & 8 & $7.70 \pm 1.40^{*}$ & $47.57 \pm 13.98^{\star}$ & $0.162 \pm 0.023^{\star}$ \\
Control group & 8 & $7.57 \pm 1.04^{*}$ & $49.06 \pm 4.46^{*}$ & $0.154 \pm 0.017^{\star}$ \\
VDL group & 8 & $3.58 \pm 0.56^{\Delta}$ & $59.15 \pm 2.51^{\Delta}$ & $0.061 \pm 0.008^{\Delta}$ \\
VDM group & 8 & $2.70 \pm 0.78^{\Delta \Delta}$ & $59.26 \pm 2.23^{\Delta}$ & $0.046 \pm 0.013^{\Delta \Delta}$ \\
VDH group & 8 & $5.27 \pm 0.30$ & $53.85 \pm 1.97$ & $0.098 \pm 0.003^{\Delta}$ \\
\hline
\end{tabular}

${ }^{*}$ Compared with normal group $(P<0.05)$, ${ }^{\Delta}$ compared with control group $(P<0.05)$, ${ }^{\Delta}$ comparison with low-dose group $(P<0.05)$. For abbreviations, see legend to Table 1.

Table 3. Levels of IL-13, OVAslgE, and IL-12 in serum of rats in each group (means \pm SD).

\begin{tabular}{llcll}
\hline Group & $\mathrm{N}$ & $\mathrm{IL}-12(\mathrm{pg} / \mathrm{mL})$ & $\mathrm{IL}-13(\mathrm{pg} / \mathrm{mL})$ & $\mathrm{OVAslgE}(\mathrm{ng} / \mathrm{mL})$ \\
\hline Normal group & 8 & $144.45 \pm 41.89$ & $33.81 \pm 4.12$ & $120.81 \pm 25.21$ \\
VD - no allergy group & 8 & $145.41 \pm 37.94$ & $31.47 \pm 6.27$ & $122.26 \pm 24.37$ \\
Model group & 8 & $70.87 \pm 5.68^{*}$ & $53.50 \pm 5.94^{*}$ & $421.02 \pm 65.10^{*}$ \\
Control group & 8 & $80.73 \pm 25.66^{*}$ & $54.43 \pm 10.28^{*}$ & $436.74 \pm 42.14^{*}$ \\
VDL group & 8 & $99.10 \pm 17.13$ & $45.80 \pm 5.30^{\Delta}$ & $289.97 \pm 55.11^{\Delta}$ \\
VDM group & 8 & $122.87 \pm 25.89^{\Delta}$ & $38.25 \pm 3.78^{\Delta \Delta}$ & $191.28 \pm 40.23^{\Delta \star}$ \\
VDH group & 8 & $46.68 \pm 19.00$ & $58.08 \pm 8.29$ & $416.17 \pm 137.98$ \\
\hline
\end{tabular}

${ }^{*}$ Compared with normal group $(P<0.05),{ }^{\Delta}$ compared with control group $(P<0.05)$, ${ }^{\star}$ comparison with low-dose group $(P<0.05)$. For abbreviations, see legend to Table 1.

Table 4. Comparison of IL-4, INF- $y$, and IL-4/INF- $y$ in bronchoalveolar lavage fluid of rats in each group (means \pm SD).

\begin{tabular}{lcccc}
\hline Group & $\mathrm{N}$ & $\mathrm{IL}-4(\mathrm{pg} / \mathrm{mL})$ & $\mathrm{INF}-\mathrm{Y}(\mathrm{pg} / \mathrm{mL})$ & $\mathrm{IL}-4 / \mathrm{INF}-\mathrm{Y}$ \\
\hline Normal group & 8 & $0.51 \pm 0.30$ & $247.36 \pm 189.18$ & $0.003 \pm 0.003$ \\
VD - no allergy group & 8 & $1.18 \pm 0.43$ & $244.07 \pm 127.98$ & $0.006 \pm 0.003$ \\
Model group & 8 & $99.97 \pm 49.62^{*}$ & $39.62 \pm 12.69^{*}$ & $2.90 \pm 2.48^{*}$ \\
Control group & 8 & $102.92 \pm 54.61^{*}$ & $43.82 \pm 13.76^{*}$ & $2.35 \pm 2.16^{*}$ \\
VDL group & 8 & $8.64 \pm 4.07^{\Delta}$ & $81.32 \pm 17.07$ & $0.11 \pm 0.05^{\Delta}$ \\
VDM group & 8 & $3.10 \pm 1.28^{\Delta}$ & $86.50 \pm 14.26$ & $0.036 \pm 0.016^{\Delta}$ \\
VDH group & 8 & $33.67 \pm 8.10^{\Delta}$ & $59.89 \pm 34.17$ & $0.76 \pm 0.44^{\Delta}$ \\
\hline
\end{tabular}

${ }^{*} \mathrm{~N}=8$, compared with normal group $(\mathrm{P}<0.05),{ }^{\Delta}$ compared with control group $(\mathrm{P}<0.05),{ }^{\wedge}$ comparison with low-dose group $(\mathrm{P}<0.05)$. For abbreviations, see legend to Table 1.

Genetics and Molecular Research 14 (4): 14136-14143 (2015)～ＯFUNPEC-RP www.funpecrp.com.br 
Table 5. Comparison of IL-12, OVAslgE, and IL-13 in bronchoalveolar lavage fluid of rats in each group (means \pm SD).

\begin{tabular}{|c|c|c|c|c|}
\hline Group & $\mathrm{N}$ & IL-12 (pg/mL) & IL-13 (pg/mL) & OVAslgE (ng/mL) \\
\hline Normal group & 8 & $35.98 \pm 10.11$ & $32.97 \pm 5.20$ & $127.95 \pm 33.33$ \\
\hline VD - no allergy group & 8 & $35.09 \pm 9.99$ & $33.14 \pm 6.48$ & $112.37 \pm 21.43$ \\
\hline Model group & 8 & $16.64 \pm 3.53^{*}$ & $74.81 \pm 4.89^{\star}$ & $354.68 \pm 77.12^{*}$ \\
\hline Control group & 8 & $20.16 \pm 6.62^{*}$ & $79.19 \pm 11.66^{*}$ & $367.84 \pm 53.03^{*}$ \\
\hline VDL group & 8 & $21.84 \pm 3.90$ & $60.04 \pm 6.63^{\Delta}$ & $235.76 \pm 54.46^{\Delta}$ \\
\hline VDM group & 8 & $26.90 \pm 5.41^{\Delta}$ & $50 \pm 8.09^{\Delta \Delta}$ & $144.72 \pm 49.34^{\Delta}$ \\
\hline VDH group & 8 & $14.44 \pm 2.94$ & $77.66 \pm 7.79$ & $362.11 \pm 119.49$ \\
\hline
\end{tabular}

${ }^{*}$ Compared with normal group $(P<0.05),{ }^{\Delta}$ compared with control group $(P<0.05),{ }^{\Delta}$ comparison with low-dose group $(P<0.05)$. For abbreviations, see legend to Table 1.

\section{Effect of vitamin D on proteins related to the IL-4/STAT6/SOCS5 signaling pathway}

Western blot showed no differences in the expressions of JAK1, STAT4, and STAT6. The expression of p-JAK1/JAK1 and p-STAT6/STAT6 was lower in the middle- and low-dose vitamin D groups than in the asthma group; their expression was enhanced in the high-dose vitamin D group, compared with that of the middle- and low-dose vitamin D groups. The expression of p-STAT4/ STAT4, p-STAT4/p-STAT6, and SOCS5 was higher in the middle- and low-dose vitamin D groups than in the asthma group; their expression was decreased in the high-dose vitamin $D$ group, compared with that of the middle- and low-dose vitamin D groups (Figure 2).

\section{Control asthma VDL VDM VDH}
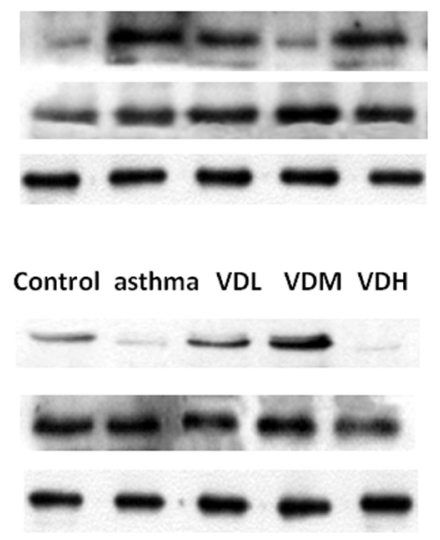

p-JAK1

JAK1

GAPDH

p-STAT4

STAT4

GAPDH
Control asthma VDL VDM VDH

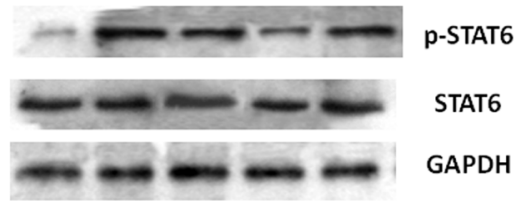

Control asthma VDL VDM VDH

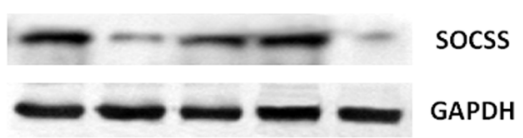

Figure 2. Expression of JAK1, p-JAK1, STAT6, p-STAT6, STAT4, p-STAT4, and SOCS5 in lung tissue of rats, as detected by western blots. For abbreviations, see legend to Table 1.

\section{DISCUSSION}

Bronchial asthma is a chronic airway inflammatory disease (van Rensen et al., 2009; Yoon, 2014). Asthma and airway inflammation are closely related and different types of airway inflammations occur in asthma. Finding of Taher et al. (2008) indicated that $1,25(\mathrm{OH})_{2} \mathrm{D} 3$ is the most important active metabolite of vitamin $\mathrm{D}$, which has a complex role in regulating chronic airway inflammation, autoimmune diseases, and chemokine expression. In this study, we elucidated 
the evaluations of in vivo anti-asthma effects when treated with different doses of vitamin $D$ and validation of the relationship between vitamin $\mathrm{D}$ and asthma. Our results revealed that middle- and low-dose vitamin $\mathrm{D}$ could alleviate inflammation in the lung tissues and could play a protective role in asthma. They reduced the IL-4, IL-13, OVAsIgE, and IL-4/INF-y levels, enhanced the IL-12 and INF-y levels in supernatants of blood and BALF, corrected the imbalanced Th1/Th2, up-regulated the expressions of proteins related to the STAT6 signal pathway, and down-regulated the expressions of proteins related to the STAT6/SOCS5 signal pathway. However, high-dose vitamin D enhanced IL-4 in the supernatants of BALF, IL-4/INF- $\gamma$ in the supernatants of blood and BALF (Varner, 2002), and worsened the imbalanced Th1/Th2, indicating that high-dose vitamin D worsened asthma, and these more serious pathological changes may be related to IL-4 and STAT6/SOCS5.

In summary, intervention with an appropriate dose of $1,25(\mathrm{OH})_{2} \mathrm{D} 3$ in early life could improve pulmonary function and reduce eosinophil cell infiltration in the airway of the rat asthma model while an overdose might have detrimental effect. Its mechanism may correlate with the effect of $1,25(\mathrm{OH}) 2 \mathrm{D} 3$ on IL-10 secreting and the expression of ICAM-1.

\section{Conflicts of interest}

The authors declare no conflict of interest.

\section{REFERENCES}

Adorini L (2002). Immunomodulatory effects of vitamin D receptor ligands in autoimmune diseases. Int. Immunopharmacol. 2: 1017-1028.

Camargo Jr CA, Rifas-Shiman SL, Litonjua AA, Rich-Edwards JW, et al. (2007). Maternal intake of vitamin D during pregnancy and risk of recurrent wheeze in children at 3 y of age. Am. J. Clin. Nutr. 85: 788-795.

Chambers ES, Suwannasaen D, Mann EH, Urry Z, et al. (2014). 1-Alpha,25-dihydroxyvitamin D3 in combination with transforming growth factor-beta increases the frequency of Foxp3(+) regulatory $\mathrm{T}$ cells through preferential expansion and usage of interleukin-2. Immunology 143: 52-60.

Devereux G, Litonjua AA, Turner SW, Craig LC, et al. (2007). Maternal vitamin D intake during pregnancy and early childhood wheezing. Am. J. Clin. Nutr. 85: 853-859.

Erkkola M, Kaila M, Nwaru BI, Kronberg-Kippila C, et al. (2009). Maternal vitamin D intake during pregnancy is inversely associated with asthma and allergic rhinitis in 5-year-old children. Clin. Exp. Allergy 39: 875-882.

He X, Wu W, Lu Y, Guo Y, et al. (2014). Effect of interleukin-33 on Th1/Th2 cytokine ratio in peripheral lymphocytes in asthmatic mice. Chin. Med. J. 127: 1517-1522.

Hiemstra PS, McCray PB Jr and Bals R (2015). The innate immune function of airway epithelial cells in inflammatory lung disease. Eur. Respir. J. 45: 1150-1162.

Liu Z, Liu X, Sang L, Liu H, et al. (2015). Boswellic acid attenuates asthma phenotypes by downregulation of GATA3 via pSTAT6 inhibition in a murine model of asthma. Int. J. Clin. Exp. Pathol. 8: 236-243.

Martin E, O'Sullivan B, Low P and Thomas R (2003). Antigen-specific suppression of a primed immune response by dendritic cells mediated by regulatory $T$ cells secreting interleukin-10. Immunity 18: 155-167.

Matheu V, Back O, Mondoc E and Issazadeh-Navikas S (2003). Dual effects of vitamin D-induced alteration of TH1/TH2 cytokine expression: enhancing IgE production and decreasing airway eosinophilia in murine allergic airway disease. J. Allergy Clin. Immunol. 112: 585-592.

Pelaia G, Vatrella A, Busceti MT, Gallelli L, et al. (2015). Cellular mechanisms underlying eosinophilic and neutrophilic airway inflammation in asthma. Mediat. Inflamm. 2015: 879783.

Taher YA, van Esch BC, Hofman GA, Henricks PA, et al. (2008). 1-Alpha,25-dihydroxyvitamin D3 potentiates the beneficial effects of allergen immunotherapy in a mouse model of allergic asthma: role for IL-10 and TGF-beta. J. Immunol. 180: 5211-5221.

Tian WM, Yang YG, Shang YX, Cai XX, et al. (2014). Role of 1,25-dihydroxyvitamin D3 in the treatment of asthma. Eur. Rev. Med. Pharmacol. Sci. 18: 1762-1769.

Truyen E, Coteur L, Dilissen E, Overbergh L, et al. (2006). Evaluation of airway inflammation by quantitative Th1/Th2 cytokine 
mRNA measurement in sputum of asthma patients. Thorax 61: 202-208.

Van Overtvelt L, Lombardi V, Razafindratsita A, Saint-Lu N, et al. (2008). IL-10-inducing adjuvants enhance sublingual immunotherapy efficacy in a murine asthma model. Int. Arch. Allergy Immunol. 145: 152-162.

van Rensen EL, Evertse CE, van Schadewijk WA, van Wijngaarden S, et al. (2009). Eosinophils in bronchial mucosa of asthmatics after allergen challenge: effect of anti-IgE treatment. Allergy 64: 72-80.

Varner AE (2002). The increase in allergic respiratory diseases: survival of the fittest? Chest 121: 1308-1316.

Yoon HI (2014). Respiratory review of 2014: asthma. Tuberc. Respir. Dis. 77: 237-242.

Zhou Y, Wang GF, Yang L, Liu F, et al. (2015). Treatment with 1,25(OH)2D3 induced HDAC2 expression and reduced NFkappaB p65 expression in a rat model of OVA-induced asthma. Braz. J. Med. Biol. Res. 48: 654-664. 